\title{
Development of Molecular Markers for Low Raffinose and Stachyose in Korean Soybean Cultivars
}

\author{
Kiwoung Yang ${ }^{1}$, Jong-Min $\mathrm{Ko}^{2}$, Tae Joung $\mathrm{Ha}^{2}$, Yeong-Hoon Lee ${ }^{2}$, In-Youl Baek ${ }^{2}$, Tae-Jin Yang ${ }^{3}$, Ill-Sup Nou ${ }^{1 *}$ \\ ${ }^{1}$ Department of Horticulture, Sunchon National University, Suncheon, Korea \\ ${ }^{2}$ Department of Function Crop, National Institute of Crop Science, Rural Development Administration, 1085 Neidong, Miryang, \\ Korea \\ ${ }^{3}$ Department of Plant Science, Plant Genomics and Breeding Institute, and Research Institute for Agriculture and Life Sciences, \\ College of Agriculture and Life Sciences, Seoul National University, Seoul, Korea
}

\begin{abstract}
A novel allele of the putative soybean raffinose synthase gene, $R S 2$, was discovered in PI200508 that is associated with the low raffinose and stachyose content. Soybean line PI200508 was identified as expressing reduced levels of raffinose and stachyose as well as elevated levels of sucrose. The RS2 mutant gene shows three base pairs InDel with the normal gene. Based on InDel region we developed novel co-dominant and dominant marker. The aim of this study was to develop Korean soybean cultivars, Daewon, Cheongja, and Danmiput, containing low levels of raffinose and stachyose. A specific markers assay for the PI200508 RS2 allele was developed to allow direct selection of the low raffinose and stachyose phenotype. Our findings highlight the efficiency of allele-specific markers in selection, which is evident in the matching genotype and results of the HPLC in the $\mathrm{F}_{2}$ generations of Daewon $\times$ PI200508 population.
\end{abstract}

Keywords HPLC analysis, Korean soybean, Low raffinose and stachyose, Molecular marker, SNP and InDel

\section{INTRODUCTION}

Soybean (Glycine $\max$ L.) is a major crop that is harvested for its protein and oil. The carbohydrate components of soybean meal or seed are composed of three major oligosaccharides: sucrose, raffinose, and stachyose (Openshaw and Hadley 1978). The respective content of these oligosaccharides in Williams 82 soybean seed generally ranges from 3.51 to $5.05 \%, 0.85$ to $1.13 \%$, and 4.65 to $5.99 \%$ (Dierking and Bilyeu 2009; Hymowitz et al. 1972). Among the three oligosaccharides, only sucrose is nutritionally useful and can be digested by monogastric animals. Raffinose and stachyose are considered nonnutritional units since the absence of a-galactosidase activity in the small and large intestines of monogastric animals and human prevents their digestion (reviewed by Roberfroid and Slavin 2000). Therefore, to promote the function of oligosaccharides in soybean, it is necessary to reduce raffinose and stachyose content while increasing sucrose content.

Soybean line PI200508 was identified as expressing reduced levels of raffinose and stachyose as well as elevated levels of sucrose (Kerr and Sebastian 2000; Parsons et al. 2000; Hitz et al. 2002). Neus et al. (2005) reported that the vigor of backcrossed, PI200508-derived, low Raffinose Family Oligosaccharides (RFO) lines is not affected as there are no significant differences in seed quality traits, such as field emergence, seed yield, maturity, height, and fatty acid content, compared to wild-type RFO-derived lines. These characteristics are essential to improve the nutritional quality of soybean meal without affecting the agronomic performance of the improved cultivars.

The key enzyme in raffinose and stachyose bisynthesis is $R S 2$. Dierking and Bilyeu (2008) determined that the molecular genetic basis for the low RFO trait in PI200508

Received June 22, 2014; Revised June 24, 2014; Accepted June 25, 2014; Published June 30, 2014

*Corresponding author Ill-Sup Nou, nis@sunchon.ac.kr, Tel: +82-61-750-3249, Fax: +82-61-750-5389 
is a variant allele of an $R S$ gene. Skoneczka et al. (2009) performed the sequence cloning and identification of the raffinose synthase mutation $1(\mathrm{rsml})$ gene for selection of a low raffinose and stachyose-containing PI200508 type. An $R S 2$ enzyme containing three-bp deletions results in low raffinose and stachyose in soybean line PI200508.

Marker-assisted selection (MAS) has many advantages compared to phenotypic selection in breeding programs. Molecular markers can increase the efficiency of plant breeding especially for complex traits (Paterson and Tanksley 1991).

The objective of this work was to develop Korean soybean cultivars using mutation-specific modification markers for $R S 2$ and evaluate its function in relation to the low raffinose and stachyose phenotype of soybean line PI200508 (Skoneczka et al. 2009). In addition, these modification markers using to $F_{2}$ population select easily low raffinose and stachyose type, heterozygous type, and normal type.

\section{MATERIALS AND METHODS}

\section{Plant materials and Molecular analysis}

Three popular soybean cultivars that are widely grown in Korea such as Daewon, Cheongja, and Danmiput were used in this study. The low raffinose and stachyose line PI200508 was used as the donor parent. Three $\mathrm{F}_{2}$ populations derived from the crosses Daewon $\times$ PI200508, Cheongja $\times$ PI200508, and Danmiput $\times$ PI200508 were developed and 150 plants from each were used for the subsequent analyses. To design an RS2-allele specific marker, the three-bp deletions (GenBank accession No. EU651888) information were introduced into the InDel-specific primer sequence (Skoneczka et al. 2009). The single nucleotide polymorphism (SNP) and insertion and deletion (InDel) primer sequences are shown in Fig. 1a (Jeong et al. 2004; Rafalski 2002; Yang et al. 2010). The expected product sizes were 252 for SNP and 131/134 bp for InDel (Fig. 1B and C).

\section{Marker and HPLC analysis}

Young trifoliate leaves of field-grown plants were used for DNA extraction. DNA from parental lines along with each $\mathrm{F}_{2}$ individual was isolated according to the protocol described by Saghai Maroof et al. (1984). To analyze markers, primers were designed using the web-based Primer3 platform (Rozen and Skaletskyv 2000). A typical PCR reaction for SNP and InDel assay includes denaturation at $94^{\circ} \mathrm{C}$ for $5 \mathrm{~min}$, followed by 25 or 34 cycles at $94^{\circ} \mathrm{C}$ for $30 \mathrm{sec}, 65^{\circ} \mathrm{C}$ or $52^{\circ} \mathrm{C}$ for $30 \mathrm{sec}, 72^{\circ} \mathrm{C}$ for $30 \mathrm{sec}$, and a final extension at $72^{\circ} \mathrm{C}$ for $7 \mathrm{~min}$. SNP product analysis was
(A) $R S 2$ (Williams 82) RS 2 (PI200508)

\author{
RS2 InDel-F RS2 SNP-F
}

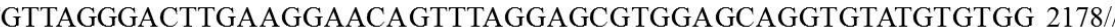
2126 TTGTTAGGGACTTGAAGGAACAGTTTAGGAGCGTGGAGCAGGTGTATGTG --- 2175//

RS2 InDel-R 2237 CTAAGGTTGTCACTCCGAAG // 2382 ATCTGCGGGTATTGACGGTGTTAA 2405

2234 CTAAGGTTGTCACTCCGAAG // 2379 ATCTGCGGGTATTGACGGTGTTAA 2402

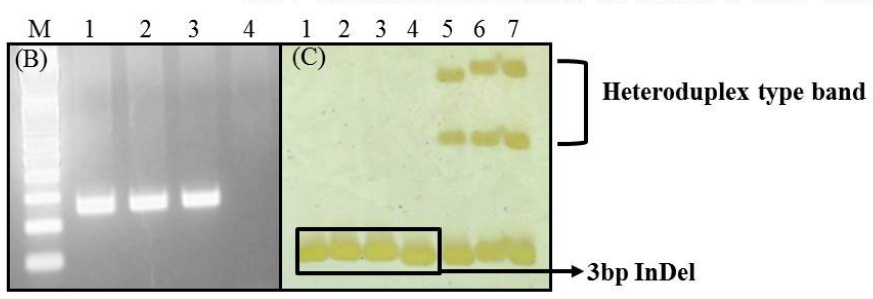

Fig. 1. Genotyping for $R S 2$ allele in Korean soybean cultivars and PI200508. (A) Nucleotide sequence (GenBank accession: EU651888) comparison of RS2 sequence in three-bp deletion to PI200508 and development of SNP and InDel marker, (B) Genotyping using the SNP type marker on agarose gel; 1: Daewon, 2: Cheongja, 3: Danmiput, 4: PI200508 (C) Genotyping using the InDel marker on polyacrylamide gel; 1: Daewon, 2: Cheongja, 3: Danmiput, 4: PI200508, 5: Daewon+PI200508 (DNA mixture), 6: Cheongja+PI200508 (DNA mixture), 7: Danmiput+PI200508 (DNA mixture). 
carried out by fragment size analysis on $1.2 \%$ agarose gel electrophoresis. PCR products for InDel were resolved on $6 \%$ polyacrylamide gel and visualized using a silver staining kit as described by the manufacturer (Promega, USA).

The $F_{2}$ seed used for determination of oligosaccharide content were simultaneously assayed for the presence of the allele, and for this, $28 \mathrm{~F}_{2}$ plants in Daewon $\times$ PI200508 population were used. Soybean oligosaccharide content were analyzed using the High-Performance Liquid Chromatography (HPLC) method (Cicek et al. 2006; Skoneczka et al. 2009).

\section{RESULTS}

\section{Allele-specific functional markers effectively select low raffinose and stachyose soybean cultivar}

The GenBank database search provides information on an annotated RS2 gene (GenBank accession EU651888) from Glycine max. Comparison of the RS2 sequences between Williams 82 and PI200508 revealed a three-bp deletion from position 2,176 to 2,178 in the predicted coding sequence (Skoneczka et al. 2009). PI200508 lacked the TGG codon (Trp) in its $R S 2$ sequence. Since a three-bp difference can be readily observed via PCR fragmentlength analysis, InDel and SNP were exploited to design a gene/mutation-specific marker for screening purposes. The sequence polymorphism between PI200508 and Korean soybean cultivars (Daewon, Cheongja, and Danmiput) RS2 alleles was used to develop modification molecular marker assays specific to the PI200508 version of the RS2 allele (Fig. 1). Further, Daewon+PI200508 (DNA mixture), Cheongja+PI200508 (DNA mixture), and Danmi+PI200508 (DNA mixture) were used to select a heteroduplex type to be used as template mixture DNA for PCR (Fig. 1C) (Jung et al. 2014). The InDel marker was used for easy identification of a heterozygous type in the populations (Fig. 2).

\section{Segregation ratio of three populations}

To improve the selection efficiency of PI200508, RS2 allele-specifc molecular markers were used to genotype the

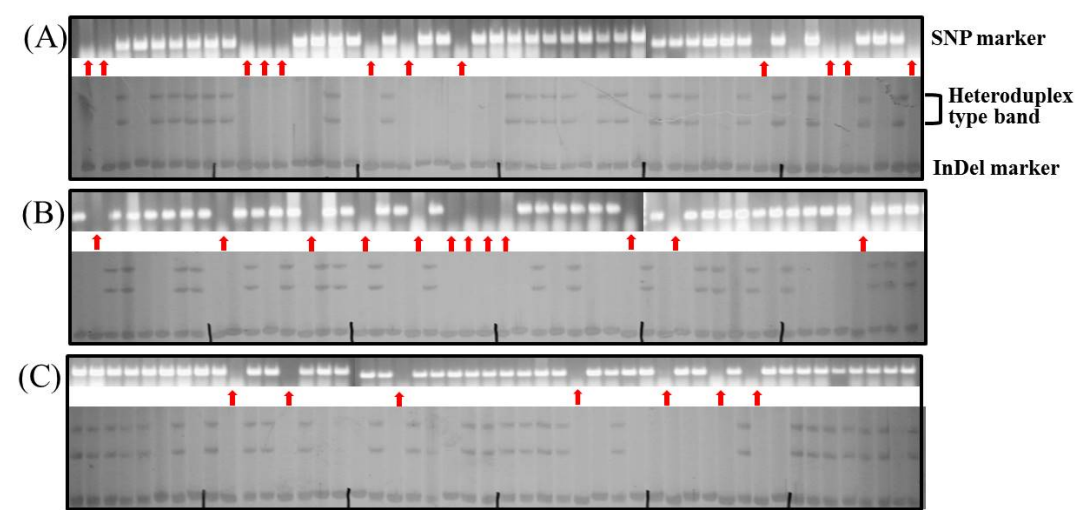

Fig. 2. Banding patterns of $R S 2$ genotype in three $\mathrm{F}_{2}$ populations assayed using SNP and InDel markers. Red arrow was selected PI200508-type. (A) Daewon×PI200508, (B) Cheongja $\times$ PI200508, (C) Danmiput $\times$ PI200508.

Table 1. Chi-square values of three $\mathrm{F}_{2}$ soybean populations segregating for low raffinose and stachyose in PI200508. Three populations show significant deviation from the expected SNP marker 3:1 wild type: PI200508 type ratio and InDel marker 1:2:1, wild type: heterozygous type: PI200508 type ratio.

\begin{tabular}{ccccc}
\hline \hline & \multicolumn{4}{c}{ Populations } \\
\hline & Daewon $\times$ PI200508 & Cheongja $\times$ PI200508 & Danmi $\times$ PI200508 & Expected ratio \\
\hline$F_{2}$ numbers & 150 & 150 & 150 & \\
SNP $(\mathrm{A}$ and $\mathrm{H}: \mathrm{B})$ & $116: 34(3.4: 1)$ & $112: 38(3.1: 1)$ & $116: 34(3.4: 1)$ & $3: 1$ \\
InDel $(\mathrm{A}: \mathrm{H}: \mathrm{B})$ & $45: 71: 34(1.3: 2.1: 1)$ & $40: 72: 38(1.05: 1.9: 1)$ & $28: 88: 34(1: 3.1: 1.2)$ & $1: 2: 1$ \\
\hline
\end{tabular}


$\mathrm{F}_{2}$ plants of Daewon $\times \mathrm{PI} 200508$, Cheongja $\times$ PI200508, and Danmiput $\times$ PI200508 segregating populations (Table 1). The data for $R S 2$ from $\mathrm{F}_{2}$ plants revealed a 1:2:1 ratio for wild-type alleles: heterozygous: PI200508 alleles, which was expected for a segregating $F_{2}$ population. The three populations derived from the crosses of PI200508 with Daewon, Cheongja, and Danmiput exhibited bimodal distribution of a recessive trait whose genetic variance is under the control of a single gene, which allows easy separation of wild-type and low stachyose individuals (Table 1). The distribution of SNP markers in these populations closely followed a 3:1 segregation ratio, which matches the expected segregation ratio for a single recessive gene in $\mathrm{F}_{2}$ populations. In addition, the InDel marker distribution seen in these populations closely fit a 1:2:1 segregation ratio, which matches the expected segregation.

\section{Consistency of genotype and phenotype}

In the Daewon $\times \mathrm{PI} 200508$ population, the 28 individuals of 150 recombination inbred lines (RILs) selected through genotyping were confirmed to have the oligosaccharide phenotype by HPLC. This population was confirmed to consist three phenotypic classes: Daewon phenotype, PI200508 phenotype, and an intermediate phenotype (Table 2 and Fig. 3). The results show that the molecular and genetic basis for the low raffinose and stachyose phenotype of PI200508 was due to a variant allele of the $R S 2$ gene.
Inheritance of the Daewon $R S 2$ alleles was sufficient to produce the Daewon oligosaccharide phenotype; whereas, inheritance of the PI200508 RS2 alleles was sufficient to produce the PI200508 oligosaccharide phenotype.

\section{DISCUSSION}

Oligosaccharides are very important nutritional components of soybean seeds, which include sucrose, raffinose, and stachyose. Of these three, sucrose is the only nutritionally useful. PI200508 was identified as a putative allele of raffinose synthase gene (RS2) in soybean that is associated with the low raffinose and stachyose phenotype. Hitz et al. (2002) studied the initial characterization of PI200508 and found that seeds of PI200508, in addition to greatly reduced levels of RFOs, have 25-fold lesser raffinose synthase enzyme activity as well as elevated levels of sucrose. The key step in raffinose and stachyose biosynthesis is most likely the enzyme $R S 2$.

The soybean line PI200508 contains a three-bp deletion in the RS2 gene that lowers the raffinose and stachyose content (Skoneczka et al. 2009). Due to the ancestral soybean genome having undergone multiple, full genome duplications (Shoemaker et al. 2006), the existence of multiple $R S$ genes is likely. Line PI200508 contained a three-bp deletion in its $R S 2$ gene. Sequencing of the $R S 2$

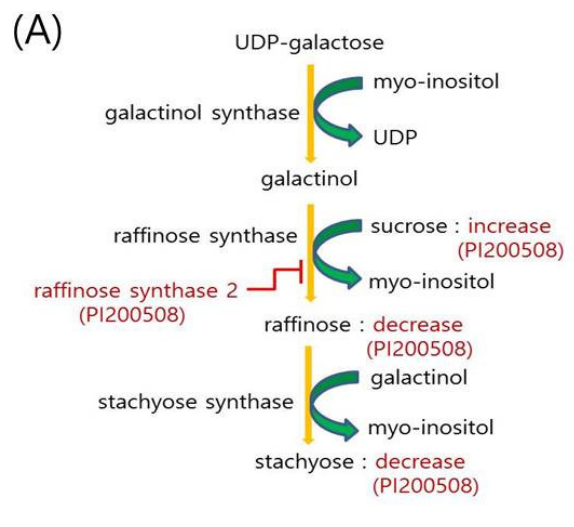

Biosynthetic pathway of RFOs in plants.
(B)

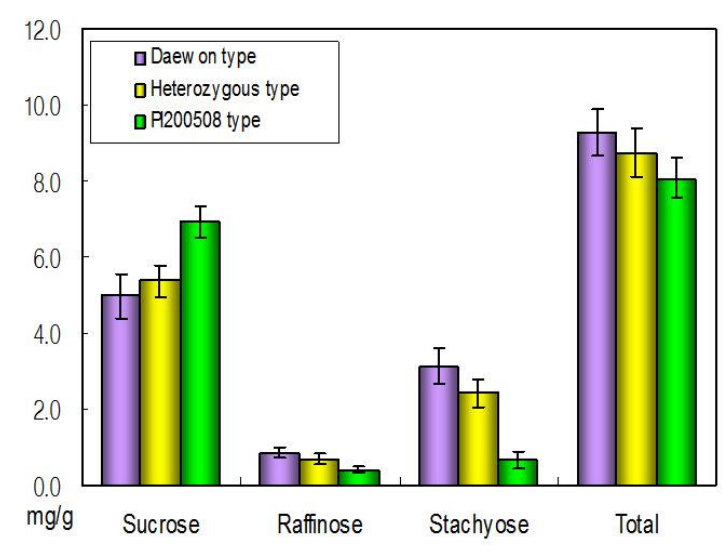

Fig. 3. Oligosaccharides content of PI200508. (A) Biosynthetic pathway of raffinose family of oligosaccharides (RFOs) in plant. Red indicates metabolism of PI200508. (B) Oligosaccharide content of seeds of selected 28 soybean plants in Daewon $\times$ PI200508 population using HPLC. 
gene from both normal cultivars as well as PI200508 revealed the polymorphisms resulting in changes of amino acid sequence. These marker assays are "breeder friendly" and allow discrimination of the PI200508 allele from the $R S 2$ alleles of other cultivars. The $R S 2$ sequence locus is located in chromosome 6 (linkage group C2) of soybean. Tissue-specific gene expression is a characteristic of a specific subset of duplicated soybean genes (Chappell et al.
2006). Effects of P1200508 allele to sucrose, raffinose, and stachylose levels in the recipient parent Korean soybean cultivars, were confirmed through the use of allele-specific molecular markers. Since PI200508 is an important source of low RFO, it can be utilized to improve the nutritional quality of soybean meal in Korea. Molecular marker assay of the Daewon $\times \mathrm{PI} 200508$ segregating population demonstrated the association of $R S 2$ genotype and the oligosaccharide

Table 2. Oligosaccharide content of 28 RILs of soybean seeds from Daewon $\times$ PI200508 population analyzed using HPLC. A: Daewon type, B: PI200508 type, Hetero: heterozygous type.

\begin{tabular}{|c|c|c|c|c|c|}
\hline Sample & Sucrose $(\mathrm{mg} / \mathrm{g})$ & Raffinose(mg/g) & Stachyose $(\mathrm{mg} / \mathrm{g})$ & Total $(\mathrm{mg} / \mathrm{g})$ & Genotype \\
\hline Daewon & 6.2 & 0.6 & 3.1 & 10.1 & A \\
\hline $912231-31$ & 4.6 & 0.7 & 3.4 & 9.0 & Homo-A \\
\hline $912231-38$ & 5.1 & 0.9 & 3.8 & 10.1 & Homo-A \\
\hline $912231-62$ & 4.6 & 0.9 & 3.3 & 9.1 & Homo-A \\
\hline $912231-64$ & 4.8 & 1.0 & 2.2 & 8.4 & Homo-A \\
\hline $912231-67$ & 5.4 & 0.8 & 3.1 & 9.6 & Homo-A \\
\hline $912231-75$ & 5.1 & 0.8 & 3.1 & 9.2 & Homo-A \\
\hline $912231-82$ & 5.1 & 0.7 & 3.5 & 9.5 & Homo-A \\
\hline 912231-103 & 4.2 & 0.8 & 3.4 & 8.6 & Homo-A \\
\hline 912231-109 & 6.3 & 1.0 & 2.9 & 10.3 & Homo-A \\
\hline $912231-112$ & 4.6 & 1.0 & 2.7 & 8.9 & Homo-A \\
\hline $912231-23$ & 5.5 & 0.7 & 3.0 & 9.3 & Hetero \\
\hline $912231-27$ & 4.7 & 0.8 & 2.5 & 8.4 & Hetero \\
\hline $912231-65$ & 5.4 & 0.8 & 2.8 & 9.3 & Hetero \\
\hline $912231-68$ & 5.8 & 0.5 & 2.4 & 8.9 & Hetero \\
\hline $912231-70$ & 5.3 & 0.5 & 2.1 & 7.9 & Hetero \\
\hline $912231-71$ & 5.2 & 0.6 & 1.8 & 7.9 & Hetero \\
\hline $912231-104$ & 5.1 & 0.7 & 2.1 & 8.1 & Hetero \\
\hline 912231-105 & 5.3 & 1.0 & 2.7 & 9.3 & Hetero \\
\hline $912231-111$ & 6.2 & 0.7 & 2.3 & 9.4 & Hetero \\
\hline $912231-14$ & 7.0 & 0.5 & 0.6 & 8.2 & Homo-B \\
\hline $912231-22$ & 7.6 & 0.5 & 0.9 & 9.1 & Homo-B \\
\hline $912231-25$ & 7.2 & 0.4 & 0.7 & 8.4 & Homo-B \\
\hline $912231-55$ & 6.6 & 0.2 & 0.5 & 7.4 & Homo-B \\
\hline $912231-58$ & 6.4 & 0.4 & 1.0 & 8.0 & Homo-B \\
\hline $912231-74$ & 6.4 & 0.4 & 0.5 & 7.3 & Homo-B \\
\hline $912231-97$ & 7.4 & 0.4 & 0.4 & 8.2 & Homo-B \\
\hline $912231-120$ & 6.9 & 0.5 & 0.8 & 8.3 & Homo-B \\
\hline $912231-140$ & 6.8 & 0.5 & 0.5 & 7.8 & Homo-B \\
\hline PI200508 & 8.3 & 0.4 & 0.9 & 9.6 & B \\
\hline
\end{tabular}


phenotype assayed through HPLC (Table 2 and Fig. 3). Using this population, matching of genotype and seed oligosaccharide phenotype was demonstrated. In summary, since the low raffinose and stachyose trait of PI200508 segregates according to a Mendelian in a dominant/recessive single gene pattern (Kerr and Sebastian 2000; Neus et al. 2005), PI200508 was crossed with three Korean soybean cultivars, Daewon, Cheongja, and Danmiput, containing normal raffinose and stachyose. Based on the findings with $\mathrm{F}_{2}$ progenies, breeding and selection for low raffinose-type can be done efficiently using allele-specific functional molecular marker.

\section{ACKNOWLEDGMENT}

This work was carried out with the support of "Cooperative Research Program for Agriculture Science \& Technology Development (Project No. PJ007155042014)" of Development of Functional Crop, National Institute of Crop Science, Rural Development Administration and Golden Seed Project (Center for Horticultural Seed Development, No. 213003- 04-2-SB110), Ministry of Agriculture, Food and Rural Affairs (MAFRA), Ministry of Oceans and Fisheries (MOF), Rural Development Administration (RDA) and Korea Forest Service (KFS).

\section{REFERENCES}

Chapell AS, Bilyeu KD. 2006. A GmFAD3A mutation in the low linolenic acid soybean line C1640. Plant Breed. 125: 535-536.

Cicek MS, Chen P, Saghai Maroof MA, Buss GR. 2006. Interrelationships among agronomic and seed quality traits in an interspecific soybean recombinant inbred population. Crop Sci. 46: 1253-1259.

Dierking EC, Bilyeu KD. 2008. Association of a soybean raffinose synthase gene with low raffinose and stachyose seed phenotype. The Plant Genome 1: 135-145.

Dierking EC, Bilyeu KD. 2009. Raffinose and stachyose metabolism are not required for efficient soybean seed germination. J. Plant Physiol. 166: 1329-1335.

DoE Joint Genome Institute. 2008. Soybean Genome Project
[Online]. Available at: http://www.phytozome.org.

Hitz WD, Carlson TJ, Kerr PS, Sebastian SA. 2002. Biochemical and molecular characterization of a mutation that confers a decreased raffinose saccharide and phytic acid phenotype on soybean seeds. Plant Physiol. 128: 650-660.

Hymowitz T, Collins F, Panczner J, Walker W. 1972. Relationship between the content of oil, protein and sugar in soybean seed. Agron. J. 64: 613-616.

Jeong SC, Saghai Maroof MA. 2004. Detection and genotyping of SNPs tightly linked to two disease resistance loci, $R s v 1$ and $R s v 3$, of soybean. Plant Breed. 123: 305-310.

Jung J, Kim KH, Yang K, Bang KH, Yang TJ. 2014. Practical application of DNA markers for high-throughput authentication of Panax ginseng and Panax quinquefolius from commercial ginseng products. J. Ginseng Res. 38: 123 129.

Kerr PS, Sebastian SA. 2000. Soybean products with improved carbohydrate composition and soybean plants. U. S. Patent 6147193. Date Issued, 14 Nov.

Neus JD, Fehr WR, Schnebly SR. 2005. Agronomic and seed characteristics of soybean with reduced raffinose and stachyose. Crop Sci. 45: 589-592.

Openshaw SJ, Hadley HH. 1978. Maternal effects on sugar content in soybean seeds. Crop Sci. 18: 581-584.

Parsons CM, Zhang Y, Araba M. 2000. Nutritional evaluation of soybean meals varying in oligosaccharide content. Poul. Sci. 79: 1127-1131.

Paterson A, Tanksley S. 1991. DNA markers in plant improvement. Adv. Agron. 46: 39-89.

Rafalski A. 2002. Applications of single nucleotide polymorphisms in crop genetics. Curr .Opin. Plant Biol. 5: 94-100.

Roberfroid M, Slavin J. 2000. Nondigestible oligosaccharides. Crit. Rev. Food Sci. Nutr. 40: 461-480.

Rozen S, Skaletskyv HJ. 2000. Primer3 on the WWW for general users and for biologist programmers, pp. 365386, In S. Krawetz and S. Misener (ed.) Bioinformatics methods and protocols: Methods in molecular biology. Humana Press Totowa NJ.

Shoemaker RC, Schlueter J, Doyle JJ. 2006. Paleopolyploidy and gene duplication in soybean and other legumes. Curr. Opin. Plant Biol. 9: 104-109.

Saghai Maroof MA, Soliman KM, Jorgensen RA, Allard RWA. 1984. Ribosomal DNA spacer-length polymorphisms 
in barley: Mendelian inheritance, chromosomal location, and population dynamics. Proc. Natl. Acad. Sci. USA 81: 8014-8018.

Skoneczka JA, Saghai Maroof MA, Shang C, Buss GR. 2009. Identification of candidate gene mutation associated with low stachyose phenotype in soybean line PI200508. Crop
Sci. 49: 247-255.

Yang K, Jeong N, Moon JK, Lee YH, Lee SH, Kim HM, Hwang CH, Back K, Palmer RG, Jeong SC. 2010. Genetic Analysis of Genes Controlling Natural Variation of Seed Coat and Flower Colors in Soybean. J.Heredity 101: 757-768. 